Check for updates

Cite this: RSC Adv., 2018, 8, 5792

Received 25th November 2017

Accepted 18th January 2018

DOI: $10.1039 / c 7 r a 12779 k$

rsc.li/rsc-advances

\section{An AuaAg nanocube based plasmonic nano-sensor for rapid detection of sulfide ions with high sensitivity $\uparrow$}

\author{
Lei Zhang, (D) *a Junxia Zhang, ${ }^{a}$ Fei Wang, ${ }^{a}$ Jingjing Shen, ${ }^{a}$ Ying Zhang, ${ }^{a}$ Lingzhi $W u,{ }^{a}$ \\ Xiaomei Lu, ${ }^{a}$ Lianhui Wang, (D) ${ }^{a}$ Quli Fan (DD *a and Wei Huang ${ }^{\text {b }}$
}

Based on the localized surface plasmon resonance (LSPR) technology, a novel plasmonic nanosensor with high sensitivity and high selectivity was prepared for the detection of trace sulfide ions on an individual Au@Ag nanoparticle. Furthermore, it could be used to monitor the sulfurization on an individual Au@Ag nanoparticle surface observed under dark-field microscopy (DFM).

\section{Introduction}

Hydrogen sulfide $\left(\mathrm{H}_{2} \mathrm{~S}\right)$, known for its toxicity, is a colorless acidic gas with a "rotten egg" smell. Recently, it was found that hydrogen sulfide is the third gaseous mediator in mammals and has an impact on human physiology and pathology, including neurotransmission, ${ }^{\mathbf{1} 2}$ vasorelaxation, ${ }^{\mathbf{3 , 4}}$ inhibition of insulin signaling, ${ }^{5,6}$ and anti-inflammation. ${ }^{7}$ Many diseases are also associated with the abnormal concentrations of $\mathrm{H}_{2} \mathrm{~S}$, such as Alzheimer's disease, ${ }^{8}$ ischaemia, ${ }^{9}$ and Down's syndrome. ${ }^{\mathbf{1 0 1 1}}$ As a new gasotransmitter similar to nitric oxide (NO) and carbon monoxide (CO), $\mathrm{H}_{2} \mathrm{~S}$ is gradually attracting more and more attention of researchers for its understanding and exploration. According to the reported results, sulfide ions could be detected now by various methods, such as the color analysis, ${ }^{\mathbf{1 2 - 1 4}}$ electrochemical analysis, ${ }^{15}$ and gas chromatography analysis. ${ }^{16}$ For example, some fluorescent $\mathrm{H}_{2} \mathrm{~S}$ probes have been designed by utilizing several chemical characteristics of $\mathrm{H}_{2} \mathrm{~S}$, such as the reduction of azide groups, ${ }^{\mathbf{1 1 , 1 2 , 1 7}}$ nucleophilic addition, ${ }^{\mathbf{1 8}}$ the $\mathrm{S}-\mathrm{Cu}$ bonding, ${ }^{19,20}$ and nucleophilic substitution. ${ }^{13,21}$ Although the limit of detection (LOD) of these methods usually only reaches $\mu \mathrm{M}$ range (which is suggested as the average endogenous $\mathrm{H}_{2} \mathrm{~S}$ level in most publications), the sulfide ion concentrations in the cellular anabolic and catabolic reactions can be as low as nM to sub-mM. ${ }^{22}$ Therefore, it is important to develop a novel nanosensor to map

${ }^{a}$ Key Laboratory for Organic Electronics and Information Displays, Institute of Advanced Materials (IAM), National Synergistic Innovation Center for Advanced Materials (SICAM), Nanjing University of Posts \& Telecommunications, 9 Wenyuan Road, Nanjing 210023, China. E-mail: iamlzhang@njupt.edu.cn; iamqlfan@njupt. edu.cn; Tel: +862585866396

${ }^{b}$ Key Laboratory of Flexible Electronics (KLOFE), Institute of Advanced Materials (IAM), National Synergistic Innovation Center for Advanced Materials (SICAM), Nanjing Tech University (Nanjing Tech), 30 South Puzhu Road, Nanjing 211816, China. Tel: +86 25 58139001

$\dagger$ Electronic supplementary information (ESI) available: Experimental details, Au@Ag NCs synthesis, immobilization of the PNPs. See DOI: 10.1039/c7ra12779k the distributions of sulfide ions and to monitor the dynamics of sulfide ion generations and eliminations inside the cell. A sensitive method with a large dynamic range means that it will be capable of following the gaseous transmitter for in-depth studies.

During the past decade, noble metal nanoparticles (NPs) have received extensive attentions for a wide variety of applications in biological sensing and imaging, ${ }^{23-25}$ catalysis, $^{26}$ photothermal therapy, ${ }^{27}$ and surface-enhanced Raman scattering (SERS), ${ }^{28,29}$ because of their unique LSPR effect. When excited by a particular frequency of incident radiation, the free electrons in the conduction band of metal nanoparticles undergo coherent oscillations, which results in their unique scattering and absorption optical phenomenon. ${ }^{25}$ LSPR characters strongly depend on the shape, size and compositions of nanoparticles and the surrounding dielectric environment. ${ }^{30,31}$ Compared to those used in fluorescent sensing, plasmonic nanomaterials used in LSPR biosensing are more sensitive, non-toxic, and do not suffer from background suppression. The LSPR scattering spectroscopy of a single plasmonic nanoparticle (PNP) could be easily performed by dark-field microscopy and a microspectral analyzer, and the notable spectral shifts indicate the refractive index of the microenvironment surrounding an individual nanoparticle. ${ }^{32,33}$

NaHS is a weak acid salt and has a dynamic equilibrium with two forms ( $\mathrm{HS}^{-}$and $\mathrm{S}^{2-}$ ) in aqueous solutions. The presence of $\mathrm{S}^{2-}$ ions and $\mathrm{Ag}$ crystals could produce a chemical reaction to form an $\mathrm{Ag}_{2} \mathrm{~S}$ precipitate. Several $\mathrm{Au}-\mathrm{Ag}$ core-shell NPs based on LSPR sensing, have been developed to detect $\mathrm{H}_{2} \mathrm{~S}$ based on this principle. $^{22,34} \mathrm{He}$ et al. have synthesized $\mathrm{Au}-\mathrm{Ag}$ core-shell nanospheres ${ }^{34}$ and used them as colorimetric probes for the recognition of $\mathrm{H}_{2} \mathrm{~S}$ through color RGB (red/green/blue) analysis of the color of PNPs. According to our group's previous results, when target miRNA-21 hybridized with probe ssDNA is immobilized on $\mathrm{Ag}$ nanocube surface, the cubic Ag nanoparticles exhibit more sensitivity to the tiny changes in the refractive index around the nanoparticle. ${ }^{35,36}$ Therefore, the Au@Ag coreshell nanocubes (NCs) exhibit a sufficient signal shift in LSPR 
peak and notable color change, which could be used as an optical plasmonic nanosensor. In this paper, a single $\mathrm{Au} @ \mathrm{Ag}$ $\mathrm{NC}$ as the sensor was prepared for the detection of trace sulfide ions by LSPR technology. We successfully monitored the process of $\mathrm{Ag}_{2} \mathrm{~S}$ formation by LSPR spectra in real time at single particle level. In the presence of different concentrations of NaHS, the peak positions in LSPR scattering spectra of individual Au@Ag sensors red shift to longer wavelengths. Moreover, there is a linear relationship between the $\Delta \lambda_{\max }$ and the sulfide ion concentrations over a large range from $\mathrm{nM}$ to $\mu \mathrm{M}$, with a lower LOD up to $0.04 \mathrm{nM}$. The high sensitivity of this probe will achieve success in broad applications in detection and real-time monitoring of trace sulfide ions in real samples.

\section{Results and discussion}

The Au@Ag NCs had better dispersions in aqueous suspensions than Ag cubes. ${ }^{35,37}$ More importantly, they had a "silver cubic sensitivity" which means that tiny changes in the surrounding environment of the nanoparticle surface will lead to more shifts in the signals of LSPR scattering spectra. As shown in Scheme 1, in the presence of sulfide ions, the activated $\mathrm{Ag}$ atoms on nanoparticle surface reacted with $\mathrm{S}^{2-}$ to generate $\mathrm{Ag}_{2} \mathrm{~S}$. There was a great difference between the refractive index of $\mathrm{Ag}(\sim 0.06)$ and $\mathrm{Ag}_{2} \mathrm{~S}(\sim 3.08)$ at $509 \mathrm{~nm},{ }^{38,39}$ which resulted in a notable redshift of the Au@Ag NC nanoparticle's scattering to a longer wavelength and a color change from cyan to dark red. On the basis of these results, Au@Ag NCs can be used as a new nanosensor to detect $\mathrm{H}_{2} \mathrm{~S}$.

$\mathrm{Au} @ A g$ NCs were prepared by the seed growth method (see $\mathrm{ESI} \dagger$ for more details). Fig. S1a and S1b $\uparrow$ are the TEM images of the Au nanospheres and Au@Ag NCs, respectively. The average size of the prepared Au@Ag NCs containing a $30 \mathrm{~nm}$ Au nanosphere core was $50.2 \pm 1.8 \mathrm{~nm}$ (see Fig. S1a, S1b and S2 $\dagger$ ). The extinction spectra of $30 \mathrm{~nm}$ Au nanospheres, Au@Ag NCs, and $\mathrm{Au} @ A g @ \mathrm{Ag}_{2} \mathrm{~S}$ NCs solutions are shown in Fig. S1c. $\dagger$ After being coated by the Ag cube, there was a blue-shift in the extinction spectrum, which exhibited the unique optical property of $\mathrm{Ag}$ NCs. ${ }^{40}$ Then, the extinction spectra red-shifted to longer wavelengths upon the formation of $\mathrm{Ag}_{2} \mathrm{~S}$ on the nanocube surface in sulfide ion solution with a certain concentration. Fig. S1d $\dagger$ illustrates the SEM image of the Au@Ag@ $\mathrm{Ag}_{2} \mathrm{~S}$ NCs which were mixed with $1 \mu \mathrm{M}$ NaHS for a certain time; sulfurization occurred at the sharp vertices and edges of NCs, which agrees with the reported results by $\mathrm{Wang}^{41}$ et al.

With the help of DFM system, the single Au@Ag NC could be employed to monitor the sulfurization process in the presence of NaHS in real time. As shown in Fig. 1a, four random Au@Ag

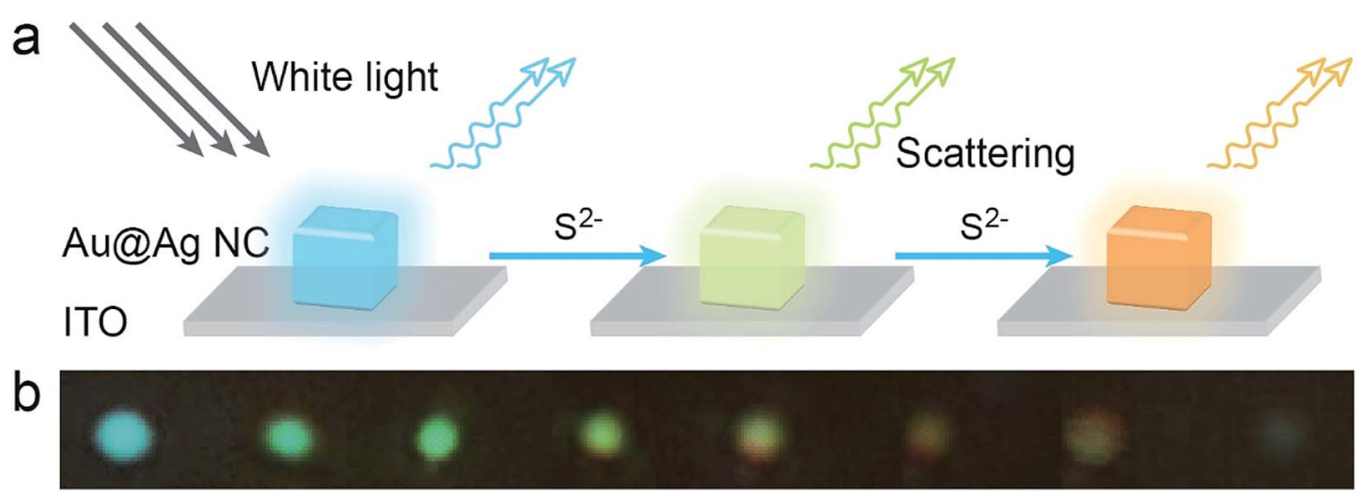

Scheme 1 (a) The scheme of sulfide ions induced color change on a single Au@Ag NC. (b) The color changes of Au@Ag NC after reacting with different concentrations of NaHS.
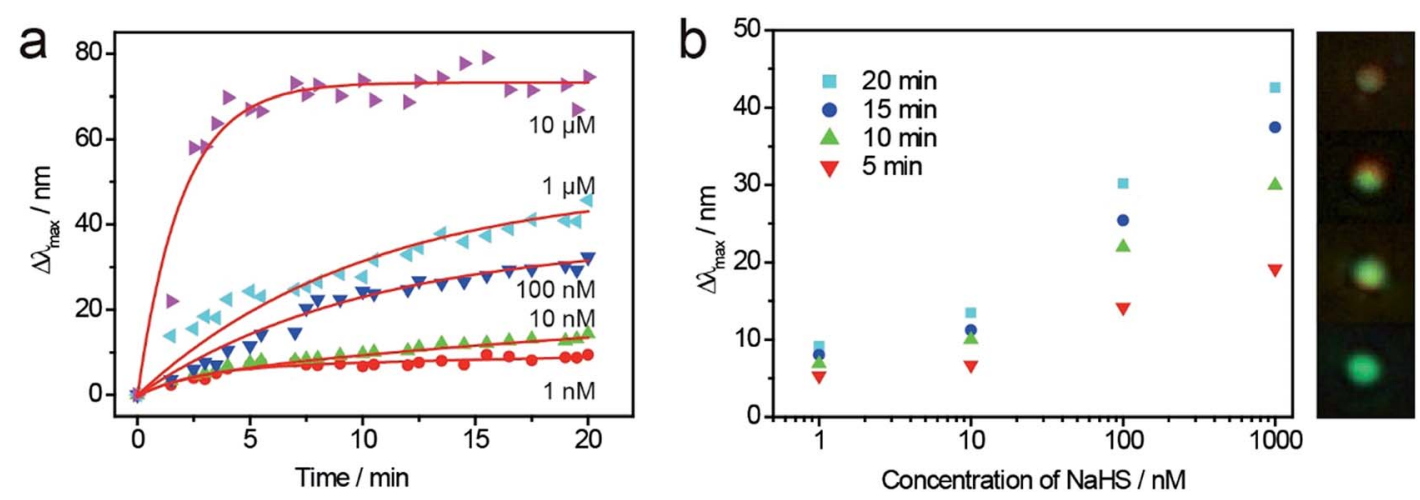

Fig. 1 (a) Measured and calculated (red lines) time-dependent spectral shifts of single Au@Ag nanoparticles exposed to concentrations of $1 \mathrm{nM}$ to $10 \mu \mathrm{M}$ NaHS. (b) Calculated $\Delta \lambda_{\max }$ values at various time points as a function of applied NaHS concentration. The illustration is the color of the particles after the reaction. 
nanosensors were selected to monitor the sulfurization process. Because of the high activity edges of NC, the peak positions of scattering spectrum red-shifted rapidly at the preliminary stage, and there was a remarkable slowdown of the reaction speed after 10 minutes. Meanwhile, the scattering peak red-shifted to a different wavelength $\left(\Delta \lambda_{\max }\right)$, which was related to the concentration of NaHS at different times (Fig. 1b). There was a good linear dependence between the peak shift and the sulfide ions concentration, which indicated that the Au@Ag NC could

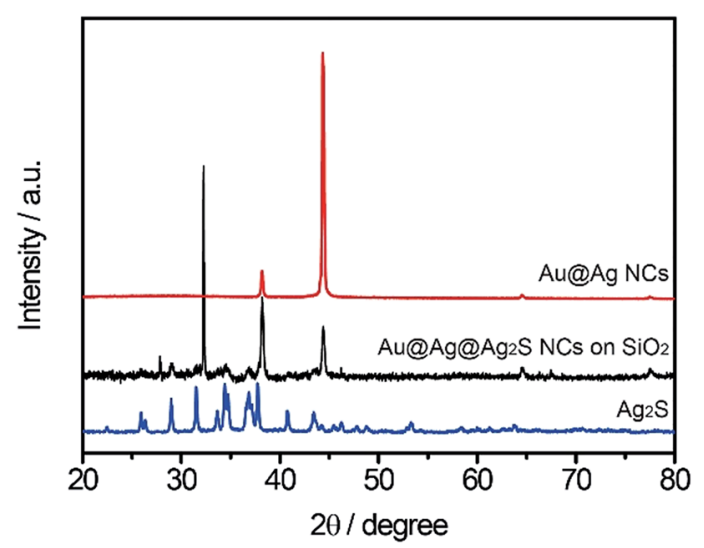

Fig. 2 XRD spectra of the Au@Ag NCs (red), Au@Ag(aAg $2 S$ (black), and $\mathrm{Ag}_{2} \mathrm{~S}$ (blue).
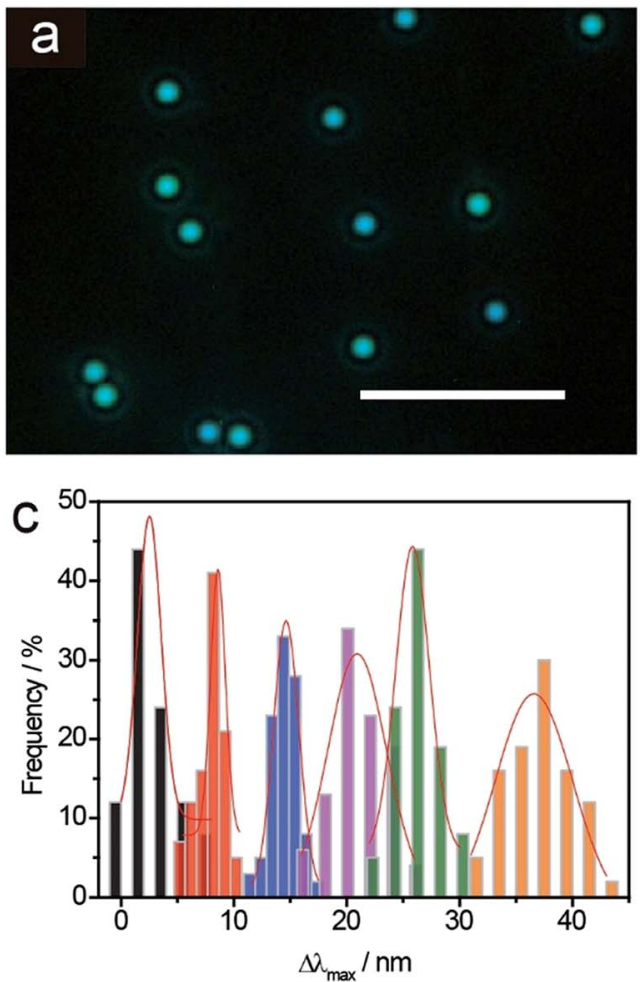

be used as a plasmonic nanosensor for the detection of sulfide ions.

Fig. 2 shows the XRD spectra of the different samples. The sharp peaks at $38-65^{\circ}$ can be assigned to (111), (200) and (220), which correspond to cubic Ag (JCPDS 89-3722). The distinctive peaks at $30-35^{\circ}$ can be assigned to (112), (121) and (022) reflections of monoclinic $\mathrm{Ag}_{2} \mathrm{~S}$ (JCPDS no. 14-0072), and they also appear in the XRD spectrum of Au@Ag@ $\mathrm{Ag}_{2} \mathrm{~S}$ NCs. These results indicate that the sulfurization process on Au@Ag NCs surface induced the peak shift of LSPR scattering spectra and color change.

To investigate the stability of the Au@Ag NCs nanosensors for detection of sulfide ions at single particle level, a large quantity of LSPR scattering experiments were repeated at the same concentration of $\mathrm{S}^{2-}$ for statistical analyses. Au@Ag NCs were immobilized on an ITO glass substrate with different concentrations of NaHS solutions for $25 \mathrm{~min}$, and their spectra and color images were obtained. Fig. 3a and b show the color change of Au@Ag NCs before and after the treatment with $1 \mu \mathrm{M}$ NaHS. During the sulfurization process, the NCs' color changed from cyan to dark red under dark-field microscopy. At the same concentration of NaHS, several LSPR nanosensors were randomly selected to observe the changes in their LSPR scattering spectra (Fig. 3c). The $\Delta \lambda_{\max }$ values were apparently linear within a large range of NaHS concentrations from $0.1 \mathrm{nM}$ to $1 \mu \mathrm{M}$ (Fig. 3d). The limit of detection was calculated to be $0.04 \mathrm{nM}$ when the ratio of $\mathrm{S} / \mathrm{N}$ was 3. Meanwhile, the probes appeared in different colors, as shown
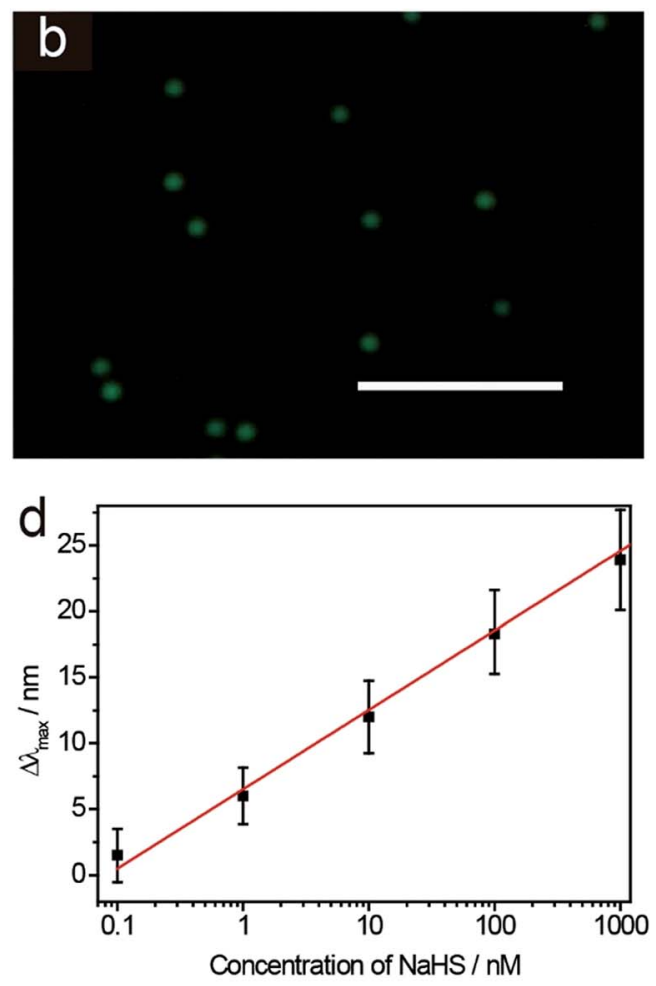

Fig. 3 Dark-field images of Au@Ag nanosensors before (a) and after (b) the treatment with $1 \mu M$ NaHS. The scale bar is $10 \mu M$. (c) Distributions of Au@Ag nanosensor LSPR peak redshift with the addition of $0.1 \mathrm{nM}$ (black); $1 \mathrm{nM}$ (red); $10 \mathrm{nM}$ (blue); $100 \mathrm{nM}$ (magenta); $1000 \mathrm{nM}$ (green); and 10 $\mu \mathrm{M}$ (orange) NaHS. Over 60 particles were counted in each case. The red lines are Gaussian distributions of maximum peak $\left(\Delta \lambda_{\text {max }}\right)$ red-shift values. (d) The line fitting curve of the average of the $\Delta \lambda_{\max }$ versus NaHS concentration. 


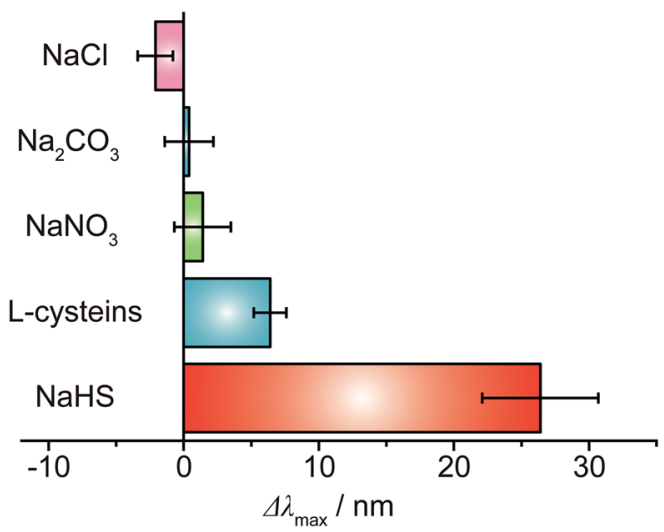

Fig. 4 Selectivity tests for sulfide ions and other anions. Legend: NaHS $(5 \mu \mathrm{M})$; L-cysteine $(50 \mu \mathrm{M}) ; \mathrm{NaNO}_{3}(50 \mu \mathrm{M}) ; \mathrm{Na}_{2} \mathrm{CO}_{3}(50 \mu \mathrm{M})$; and $\mathrm{NaCl}$ $(50 \mu \mathrm{M})$.

in Scheme $1 b$, in the corresponding concentrations of NaHS; thus, it could be used for colorimetric detection.

To verify the selectivity of Au@Ag nanosensor for detection of sulfide ions, different interfering ions (Fig. 4) were selected to perform the same experiment at the same condition. Fig. 4 shows the selectivity test results of LSPR scattering spectra shift in the presence of $\mathrm{NaCl}, \mathrm{Na}_{2} \mathrm{CO}_{3}, \mathrm{NaNO}_{3}$, L-cysteine and $\mathrm{NaHS}$, respectively. There was no remarkable color change with presents of $\mathrm{Cl}^{-}, \mathrm{CO}_{3}{ }^{2-}$ and $\mathrm{NO}_{3}{ }^{-}$, which indicated that the nanosensors could be used for trace sulfide ion detection with high selectivity. LSPR scattering spectra peak position exhibited a slight blue shift. This result might be attributed to the slight RI change around the nanosensors, caused by the damage of the bilayer of CTAB on $\mathrm{Au} @ \mathrm{Ag}$ NC surface by charged ions. Moreover, there was only 5-8 $\mathrm{nm}$ red-shift in the presence of a 10-fold concentration of $\mathrm{HS}^{-}$because of the L-cysteine absorption on the Au@Ag NC plasmonic nanosensor surface. The results indicated that this LSPR nanosensor can be used for the detection of sulfide ions with high selectivity.

\section{Conclusions}

This study introduces a new method for real-time monitoring of the sulfurization process, occurring on an individual $\mathrm{Au} @ \mathrm{Ag} \mathrm{NC}$ surface, and the LSPR scattering spectra red-shifts to longer wavelengths in the presence of different concentrations of sulfide ions. In addition, this process can be easily observed by the color change from the DFM images. These results can be used to design novel plasmonic nanosensors for detection of trace sulfide ions. There is a linear dependence between the LSPR scattering spectra red-shift and different concentrations of sulfide ions ranging from $0.1 \mathrm{nM}$ to $1 \mu \mathrm{M}$. Moreover, this plasmonic method demonstrates more sensitivity (LOD: 0.04 $\mathrm{nM}$ ) and selectivity. With the developments in LSPR, nanosensors at nano scale can be expected to detect other molecules for chemical and biological applications.

\section{Conflicts of interest}

There are no conflicts to declare.

\section{Acknowledgements}

This work was supported by the National Natural Science Foundation of China (61571239, 21475064, 21674048), the Scitech Support Plan of Jiangsu Province (BE2014719), the Program for Changjiang Scholars and Innovative Research Team in University (IRT_15R37), the Scientific Research Foundation of Nanjing University of Posts and Telecommunications (NY215173), and the Priority Academic Program Development of Jiangsu Higher Education Institutions (YX03001).

\section{Notes and references}

1 A. Salvi, P. Bankhele, J. M. Jamil, M. Kulkarni-Chitnis, Y. F. Njie-Mbye, S. E. Ohia and C. A. Opere, Neurochem. Res., 2016, 41, 1020-1028.

2 V. S. Fernandes, A. S. Ribeiro, P. Martinez, M. E. Lopez-Oliva, M. V. Barahona, L. M. Orensanz, A. Martinez-Saenz, P. Recio, S. Benedito, S. Bustamante, A. Garcia-Sacristan, D. Prieto and M. Hernandez, PLoS One, 2014, 9, e113580.

3 Y. Sun, Y. Huang, W. Yu, S. Chen, Q. Yao, C. Zhang, D. Bu, C. Tang, J. Du and H. Jin, Oncotarget, 2017, 8, 31888-31900.

4 Q. Chai, T. Lu, X. L. Wang and H. C. Lee, Pflügers Archiv, 2015, 467, 329-340.

5 N. Hu, M. Dong and J. Ren, Am. J. Physiol. Regul. Integr. Comp. Physiol., 2014, 306, R761-R771.

6 G. Tang, L. Zhang, G. Yang, L. Wu and R. Wang, Diabetologia, 2013, 56, 533-541.

7 J. L. Wallace, R. W. Blackler, M. V. Chan, G. J. Da Silva, W. Elsheikh, K. L. Flannigan, I. Gamaniek, A. Manko, L. Wang, J. P. Motta and A. G. Buret, Antioxid. Redox Signaling, 2015, 22, 398-410.

8 A. Xuan, D. Long, J. Li, W. Ji, M. Zhang, L. Hong and J. Liu, J. Neuroinflammation, 2012, 9, 202-213.

9 I. Andreadou, E. K. Iliodromitis, T. Rassaf, R. Schulz, A. Papapetropoulos and P. Ferdinandy, Br. J. Pharmacol., 2015, 172, 1587-1606.

10 H. Yang, Y. Zhang, L. Li, G. Sun, L. Zhang, S. Ge and J. Yu, Biosens. Bioelectron., 2017, 87, 53-58.

11 K. Wang, H. Peng, N. Ni, C. Dai and B. Wang, J. Fluoresc., 2014, 24, 1-5.

12 N. Adarsh, M. S. Krishnan and D. Ramaiah, Anal. Chem., 2014, 86, 9335-9342.

13 L. A. Montoya, T. F. Pearce, R. J. Hansen, L. N. Zakharov and M. D. Pluth, J. Org. Chem., 2013, 78, 6550-6557.

14 Y. Zhao, X. Zhu, H. Kan, W. Wang, B. Zhu, B. Du and X. Zhang, Analyst, 2012, 137, 5576-5580.

15 E. Bitziou, M. B. Joseph, T. L. Read, N. Palmer, T. Mollart, M. E. Newton and J. V. Macpherson, Anal. Chem., 2014, 86, 10834-10840.

16 M. Deng, M. Zhang, X. Huang, J. Ma, L. Hu, G. Lin and X. Wang, Journal of Forensic and Legal Medicine, 2015, 32, 59-63.

17 S. Chen, Z. J. Chen, W. Ren and H. W. Ai, J. Am. Chem. Soc., 2012, 134, 9589-9592.

18 C. Liu, B. Peng, S. Li, C. M. Park, A. R. Whorton and M. Xian, Org. Lett., 2012, 14, 2184-2187. 
19 L. E. Santos-Figueroa, C. de la Torre, S. El Sayed, F. Sancenón, R. Martínez-Máñez, A. M. Costero, S. Gil and M. Parra, Eur. J. Inorg. Chem., 2014, 2014, 41-45.

20 K. Sasakura, K. Hanaoka, N. Shibuya, Y. Mikami, Y. Kimura, T. Komatsu, T. Ueno, T. Terai, H. Kimura and T. Nagano, J. Am. Chem. Soc., 2011, 133, 18003-18005.

21 T. Liu, Z. Xu, D. R. Spring and J. Cui, Org. Lett., 2013, 15, 2310-2313.

22 B. Xiong, R. Zhou, J. Hao, Y. Jia, Y. He and E. S. Yeung, Nat. Commun., 2013, 4, 1708-1717.

23 J. Li, J. Liu and C. Chen, ACS Nano, 2017, 11, 2403-2409.

24 G. Doria, J. Conde, B. Veigas, L. Giestas, C. Almeida, M. Assuncao, J. Rosa and P. V. Baptista, Sensors, 2012, 12, 1657-1687.

25 P. K. Jain, X. Huang, I. H. El-Sayed and M. A. El-Sayed, Acc. Chem. Res., 2008, 41, 1578-1586.

26 K. K. Haldar, S. Kundu and A. Patra, ACS Appl. Mater. Interfaces, 2014, 6, 21946-21953.

27 B. Jang, J. Y. Park, C. H. Tung, I. H. Kim and Y. Choi, ACS Nano, 2011, 5, 1086-1094.

28 L. Pei, Y. Ou, W. Yu, Y. Fan, Y. Huang and K. Lai, J. Nanomater., 2015, 16, 1-8.

29 L. Guerrini and D. Graham, Chem. Soc. Rev., 2012, 41, 70857107.
30 J. Wirth, F. Garwe, J. Bergmann, W. Paa, A. Csaki, O. Stranik and W. Fritzsche, Nano Lett., 2014, 14, 570-577.

31 Y. Li, C. Jing, L. Zhang and Y. T. Long, Chem. Soc. Rev., 2012, 41, 632-642.

32 L. Shi, C. Jing, W. Ma, D.-W. Li, J. E. Halls, F. Marken and Y.-T. Long, Angew. Chem., Int. Ed., 2013, 52, 6011-6014.

33 R. Qian, Y. Cao and Y.-T. Long, Angew. Chem., Int. Ed., 2016, 55, 719-723.

34 J. Hao, B. Xiong, X. Cheng, Y. He and E. S. Yeung, Anal. Chem., 2014, 86, 4663-4667.

35 L. Zhang, Y. Zhang, Y. Hu, Q. Fan, W. Yang, A. Li, S. Li, W. Huang and L. Wang, Chem. Commun., 2015, 51, 294-297.

36 Y. Hu, L. Zhang, Y. Zhang, B. Wang, Y. Wang, Q. Fan, W. Huang and L. Wang, ACS Appl. Mater. Interfaces, 2015, 7, 2459-2466.

37 Q. Zhang, W. Li, C. Moran, J. Zeng, J. Chen, L. P. Wen and Y. Xia, J. Am. Chem. Soc., 2010, 132, 11372-11378.

38 P. B. Johnson and R. W. Christy, Phys. Rev. B, 1972, 6, 43704379.

39 J. M. Bennett, J. L. Stanford and E. J. Ashley, J. Opt. Soc. Am., 1970, 60, 224-231.

40 G. Park, D. Seo, J. Jung, S. Ryu and H. Song, J. Phys. Chem. C, 2011, 115, 9417-9423.

41 C. Fang, Y. H. Lee, L. Shao, R. Jiang, J. Wang and Q. H. Xu, ACS Nano, 2013, 7, 9354-9365. 